\title{
Optimization of Routing Protocolbased on K-Anycast for Efficiency Increasing and Wireless Chargeable Sensor Net Security
}

\author{
HamedAlinejad $^{1,}$ KambizMajidzadeh $^{2}$ \\ ${ }^{I}$ Software MSc. Computer department, Azad University branch of Urmia \\ ${ }^{2}$ Assistant professor, Computer department, Azad University branch of Urmia
}

\begin{abstract}
Considering that routing and its protocol is the base of digital data transmission, in this article has been tried that role of Routing Protocol based on K-Anycast has been evaluated in optimization of wireless chargeable sensor nets operation.In conventional WSNs, the source nodes select a sink among the sinks for the package delivery.But the K-anycast tries to select the neighbor nodes with the highest possible sink for optimizing the reliability, balance and security.More sensors should be used in data transmission for $K$-anycast design, that caused the energy consumption rising.These properties show that K-anycast technique is only suitable for RWSNs. Here three sketch upon to tree model that sink node is assumed as the root and sensor nodes are assumed as leaves, are assessed forK-anycast. Results show that K-anycast method show better operation than other methods.
\end{abstract}

Keywords: wireless sensor networks, anycast, Ad hoc networks, chargeable sensors, routing, $K$-anycast

\section{Introduction}

Generally, the computer networks can be implemented via two method (infrastructure and Ad-Hoc).In first kind, the network implementation needs at least one central device as an access point and the network structure generated based on standard base. In second kind, the peripheral devices do not use and each one of computers has the access point role. In this study, it has been tried to evaluate the routing protocols based on Kanycast (Wu, 2006) on optimization of wireless sensor networks. Ad-Hoc has the Latin root and means the individual application. It explains that it can be used when the other services that implemented generally cannot be utilized. Recent developments in integrated circuit generation technology in micro size in one side and wireless communication technology improvement on the other hand caused the wireless sensor networks designing. Wireless sensor networks are the especial kind of Ad-Hoc networks, which made for specific targets with specific structure. These networks installed, sat up and managed in limited domain and specific area such as military, biologic, industrial (Ganiyu, 2014), health (Hackmann, 2014), domestic (Wang, 2014), security etc. Aforementioned points show that there are challenges in this field such as security, speed, calculation accuracy, optimum routing, energy optimum consumption etc. figure one shows the standard structure and one node of wireless sensor network.

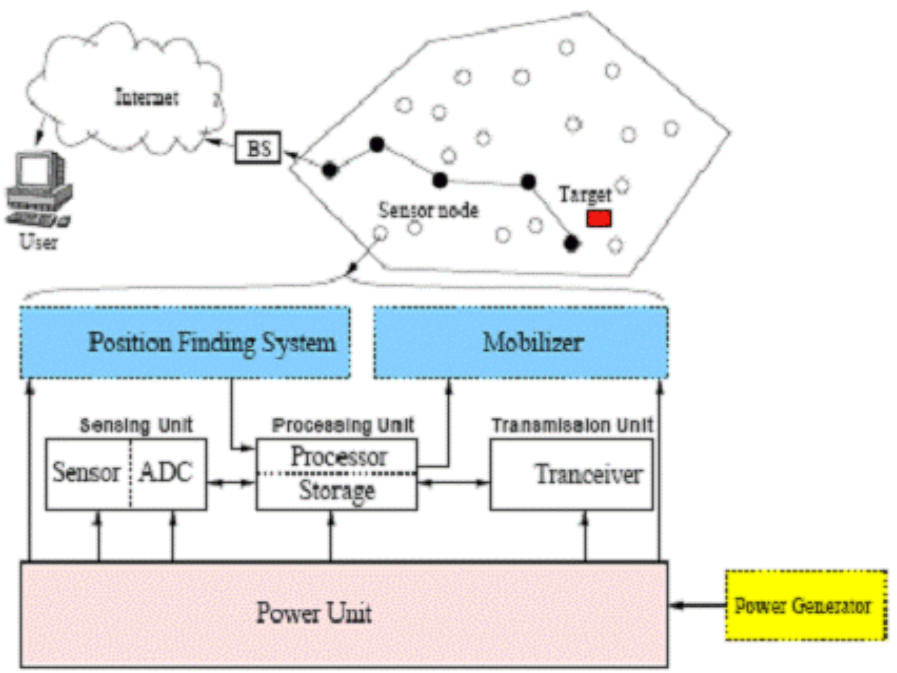

Fig1. Wireless sensor network structure and its nodes 


\section{Methods and algorithms}

The KanGuRou algorithm (Mitton, N, 2012) will be explained in present section that is an algorithm based on location and data delivery guaranty. This algorithm is the modified and plug in form of EEGPA algorithm (Mitton, N, 2012), which has presented for the energy saving in wireless sensor networks with kanycast approach as an efficient algorithm. KanGuRou is an algorithm without memory and high scaling ability. The main idea of KanGuRou is the information sending to each one of k sink among the sink aggregate (S). Table 1 shows the KanGuRou algorithm.

Table1. KanGuRou algorithm

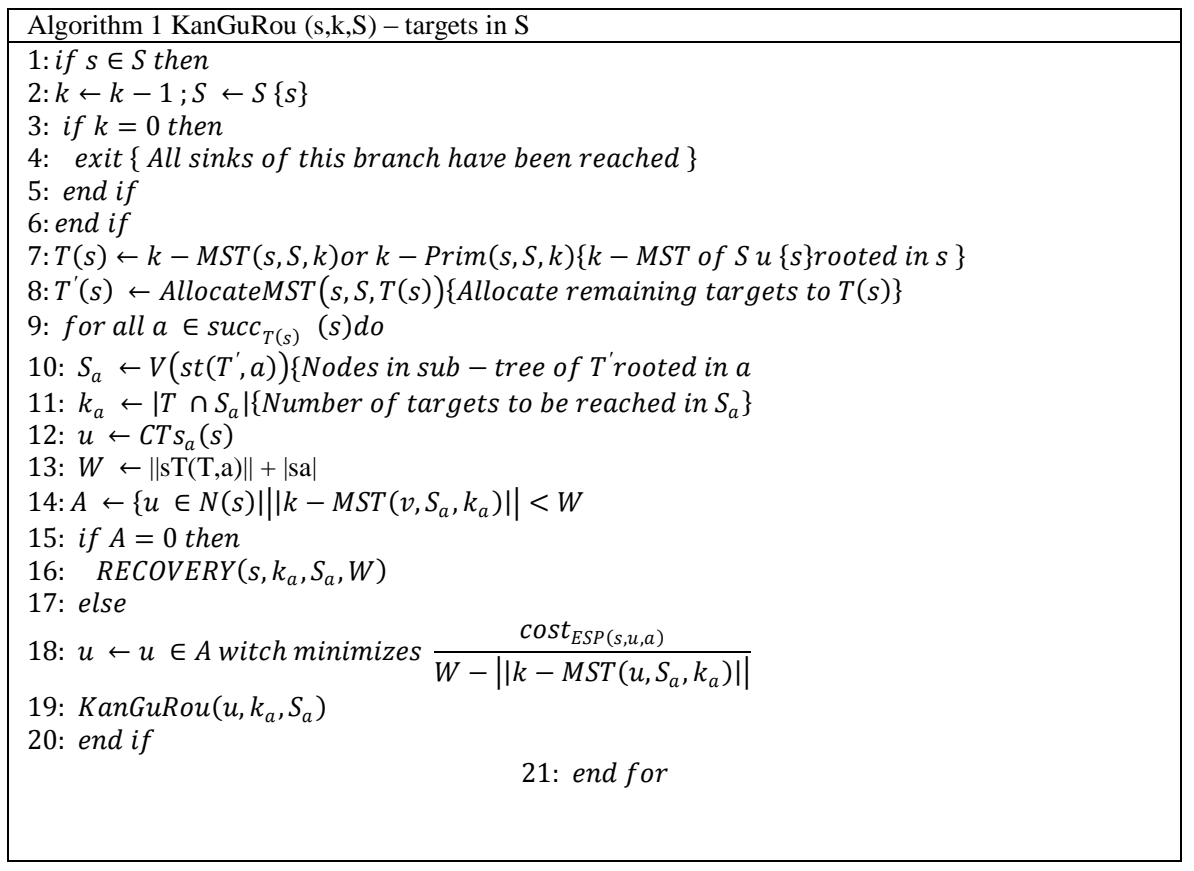

\section{Method of operation in KanGuRou}

At first the node $\mathrm{s}$ keeps the message and if itself was sink, reduces one unit of $\mathrm{k}$. if the $\mathrm{k}=0$ means that the message has been sent to all k sinks; so the algorithm will end. The s node calculates and processes the $\mathrm{T}(\mathrm{s})$ tree by one of $\mathrm{k}-\mathrm{MST}(\mathrm{s}, \mathrm{S}, \mathrm{k})$ or k-Prim$(\mathrm{s}, \mathrm{S}, \mathrm{k})$ algorithms. If there were many branches from $\mathrm{s}$, then an iteration in messaging will occur. The s nodes distributes the remained sinks, therefore for each a substitute from $\mathrm{s}$ in $\mathrm{T}(\mathrm{s})$, a subset $\mathrm{S}_{\mathrm{a}}<\mathrm{S}$ assumed to a. the branch $\mathrm{S}_{1}$ with $\mathrm{S}_{1}, \mathrm{~S}_{3}$ and $\mathrm{S}_{4}$ sinks will be assumed whereas the sinks $\mathrm{S}_{2}, \mathrm{~S}_{5}, \mathrm{~S}_{6}, \mathrm{~S}_{7}$ and $\mathrm{S}_{8}$ connect to branch $\mathrm{S}_{5}$. In this stage, the node s sends some packs per each substitute in $\mathrm{T}(\mathrm{s})$. Therefore, for each branch of $\mathrm{T}(\mathrm{s})$ per each substitutes of $\mathrm{a}$, the node $\mathrm{s}$ calculates the $\mathrm{k}_{\mathrm{a}}-\mathrm{MST}$ weight per each one of its neighbors on $\mathrm{S}_{\mathrm{a}}$ set.

$\left\|\mathrm{k}-\operatorname{MST}\left(\mathrm{u}, S_{\mathrm{a}}, k_{\mathrm{a}}\right)\right\|(1)$

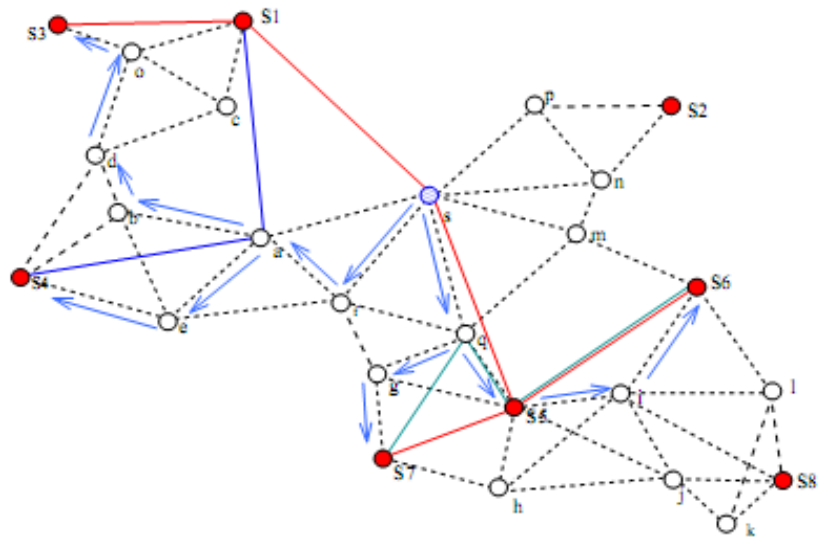

fig2. The KanGuRou algorithm implementation by k-MST (red lines) and k-Prim (blue lines) 
Based on figure 2, the node s calculates the term 3-MST on $S_{2}, S_{5}, S_{6}, S_{7}$ and $S_{8}$ sinks, finding the next step for branch $\mathrm{S}_{5}$; In addition, the 2-MST on $\mathrm{S}_{1}, \mathrm{~S}_{3}$ and $\mathrm{S}_{4}$ sinks of branch $\mathrm{S}_{1}$. If there were not any neighbor $\mathrm{u}$ for s per $\left\|\mathrm{k}-\mathrm{MST}\left(\mathrm{u}, S_{\mathrm{a}}, k_{\mathrm{a}}\right)\right\|<\|\mathrm{sT}(\mathrm{T}, \mathrm{a})\|+\left|\mathrm{S}_{\mathrm{a}}\right|$, the node s switches towards the a as recovery state.

\section{Suggested method}

A sensor node needs to generate a source tree, anycast, to destination sink for information gathering. This will be done, using the control packages based on neighboring and selection strategy. An anycast tree is generated via below steps:

1- A source starts to send PRQ to its neighbors with maximum transmission radii.

2- If the node's neighbors received the PRQ package and anycast trees joined to each other, nodes will accepted the PRQ and calculate the path cost base on PRQ information. Then these nodes send CRQ to the transmitter.

3- After the PRQ sending, the transmitter may receive more than one CRQ from its neighbors. If the empty sink id was CRQ, it shows that CRQ was from one sink and transmitter selects the node as the parent neighbor; otherwise, transmitter evaluates all CRQs.

Depends on CRQ, the transmitter selects the neighbor node with minimum path cost, $\Phi$, and the parent node via the path cost comparison in CRQs. The strategy of neighbor node selection deals with integer number programming between the zero and one. In that unit, current information record the parent node and return a PREP to the parent node. If there were more than one path cost, $\Phi$, for same CRQs, the neighbor node is selected arbitrary. The process of neighbor node selection has been shown in figure three.

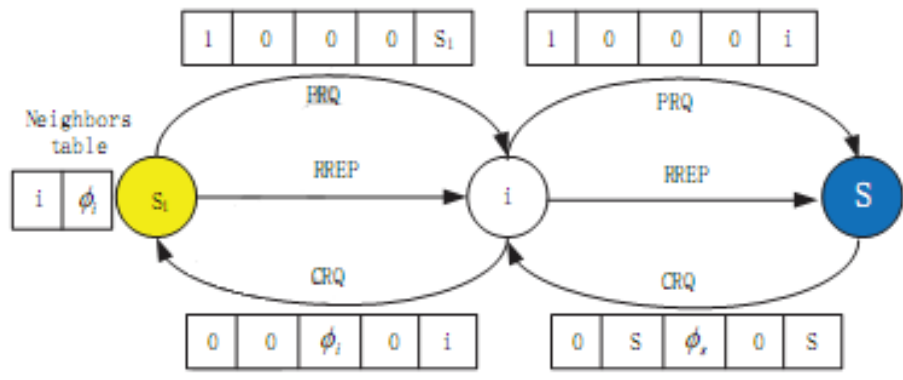

Fig3. The process of Neighbor node selection

4- After the PREP receiving by parent node, a transmission canal is generated between the transmitter and parent node. After that, the parent node keeps sending the PRQ to the neighbor nods.

Upon to algorithm properties, each node needs to save the next node information. The parent node does not need to record the related information about transmitter, which is different from TCP/IP (Forouzan, 2002). The ID transmitter replaces the next nodes in PRQ by the receiver ID. The $\Phi$ of path cost from transmitter to receiver is recalculated and the initial path cost $\Phi$, substitutes in neighbor table. New path cost now can be calculated, receiver changes to transmitter to send the PRQ in next step until it reaches to sink and shows that each tree set up successfully. Figure 4 shows that two sources make two-anycast tree to reach to sinks. After that, each node institutionalized one anycast tree to reach to the common sink and sensors.

\section{Results}

There are two challenge in wireless sensor networks: the first one is node awareness about the remained energy and second one is the limited battery capacity for recharging.

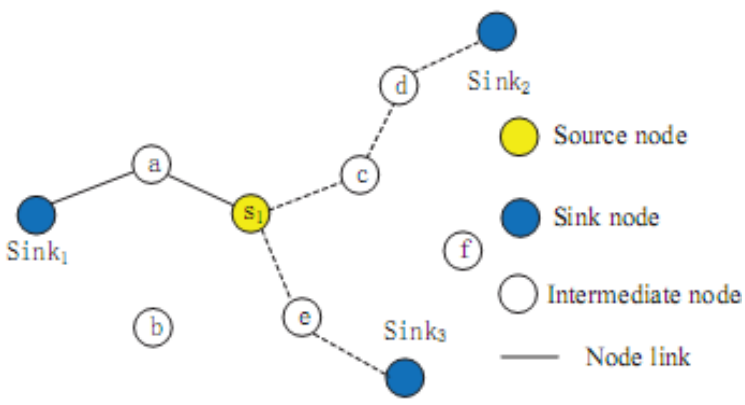

Fig4. Data sending by a sensor towards the adjacent sinks 


\section{System model}

A wireless sensor network (with $\mathrm{n}$ sensor and sink node) as graph is shown by below relation:

$\mathrm{G}=(\mathrm{V}, \mathrm{A})$

Where $\mathrm{V}$ is set of $\mathrm{n}$ nodes in network and $\mathrm{A}$ is set of link paths between nodes. $A=\{A \mid(i, j) \in A, i, j \in V\}$.

Graph $\mathrm{G}$ consists of limited nodes of $\mathrm{V}$ set and $\mathrm{A}$, containing the regular path between nodes.

\section{The strategy of sink and neighbor nodes selection}

In wireless sensor networks with more sinks, there are two challenges in information transmission. First challenge is the selection of sink from the all destination sinks for information receiving andsecond one is the neighbor node selection for information forwarding to destination. Determining the information transmission cost, finding the neighbor node, and sink the below relation is used:

$\phi=\sum_{i=1}^{i=k} a_{i} *$ Metric $_{i}$

Routing cost of two adjacent nodes will be as below:

$\phi=a_{1} * h+a_{2} * d+a_{3} * c+a_{4} * r(4)$

In this relation, the jump number for two-neighbor node equals to one. The energy cost shows the general energy consumption for the link making between two-neighbor nodes directly. If there were m node of sensors the total cost can be obtained by relation 5 :

$\Pi=\sum_{j=1}^{j=m} * \sum_{i=1}^{i=k} a_{i} *$ Metric $_{i}$

Consider a network with $\mathrm{n}$ sensor node on $\mathrm{m}$ sources and a group of sinks equal to $\mathrm{k}$ which $1 \leq \mathrm{k} \leq \mathrm{n}$. the target is assuming each source to any sink to minimize the total costs. This problem is shown as below:

$\min f(x) \quad(8)$

subjectto : $\quad(9)$

$f(x)=\sum_{i=1}^{n} \quad \sum_{j=1}^{k} \phi_{i, j} \lambda_{i, j}$

$\phi_{i, j}=a_{i} *$ Metric $_{i}, 1 \leq i \leq n, 1 \leq j \leq n$

$\lambda_{i, j}=0$ or $1,1 \leq i \leq n, 1 \leq j \leq n$

Where $\phi_{i, j}$, is the best routing cost between $\mathrm{j}$ and I.in addition, $\lambda_{i, j}$ is a variable with 0 and 1 amount for the best sink selection. If the best sink, reaching the information, moves from $\mathrm{I}$ to $\mathrm{j}$ it will be one and if it did not happen the answer will be zero.

\section{Sending maximum to $\mathrm{k}$ sink}

In information delivery process, there are two probable cases: first, may different messages follow same path and second the messages may change the targets (fig 5). These probabilities are the great challenges for the routing protocols and cause that a source node could not control the number of considered targets. When there are various packages in a same path, a line should be generated and wait for an empty canal that causes a serious delay (lag). Avoiding this flaw, in current study just a case considered messages that send to dissimilar sensors.

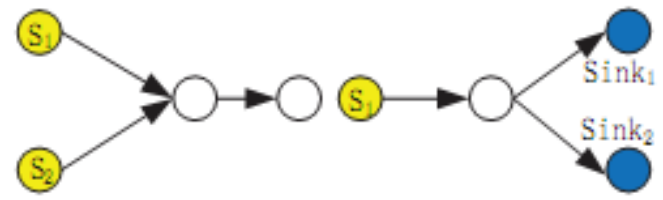

Fig5. Message transmission in same path or the sudden changes of target node

\section{Minimum link K-anycast and exact K-anycast}

To ensure about the information deliverance by the minimum or exact amount of $\mathrm{k}$ sink, the source node should deliver their information to minimum or exact $k$ number, forwarding the messages. If the $p \geq k$ then a source node can receive the answers of p neighbors.

\section{Result and discussion}

For the assessment of suggested algorithm operation, it will compared with KanGuRou (Miton 2012) and Eu EHOR (Zhi Ang 2010).

\section{System performance}

Here a path process will performed, using the anycast link pattern with 100 sensor and five sink in a $500 \mathrm{~m} * 500 \mathrm{~m}$ area. In training scenario, the source nodes generate some packages with environment sensory and sending them to $\mathrm{k}$ sinks $(\mathrm{k}=3)$. All routing path will be experimented. Considering the energy saving, the source 
Optimization of Routing Protocolbased on K-Anycast for efficiency increasing and wireless..

node selects the nearest base station as a destination. However, the all package of sources may cross all nodes before the reaching to $\mathrm{k}$ sinks to improve the sensor performance.

\section{Analyze and comparison}

At first, the average of information transmission lag in graph nodes, the KAnGuRou and EHOR methods compared, considering the $(\mathrm{a} 1, \mathrm{a} 2, \mathrm{a} 3, \mathrm{a} 4)=(0,1,0,0,0)$ as it can be seen in figure 6 .
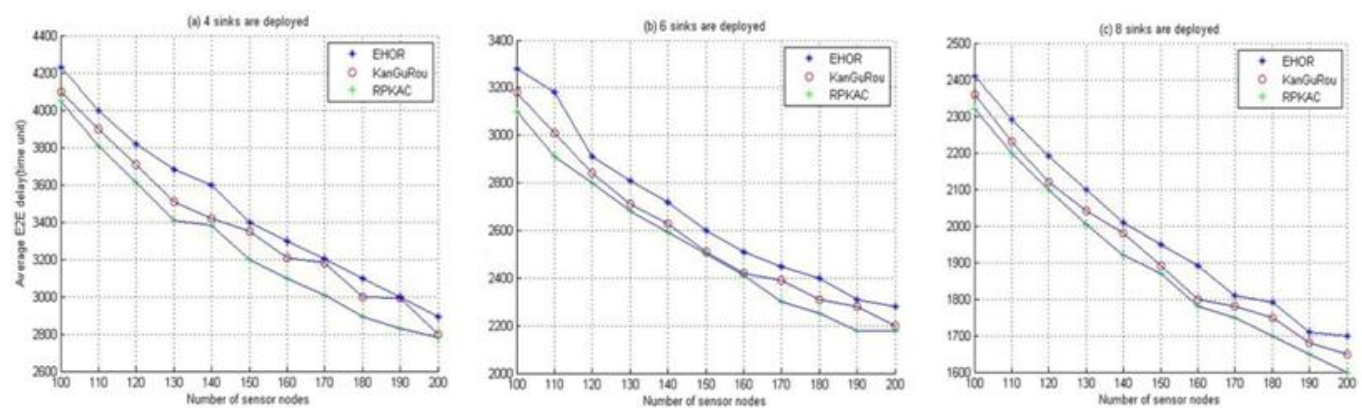

Fig6. The average lag of E2E with increasing node number

As it can be seen in figure 6, the lag time reduces, increasing the duty cycle in each three algorithms. However, the lag in suggested algorithm (RPKAC) is lesser than other two algorithms during the data transmission.
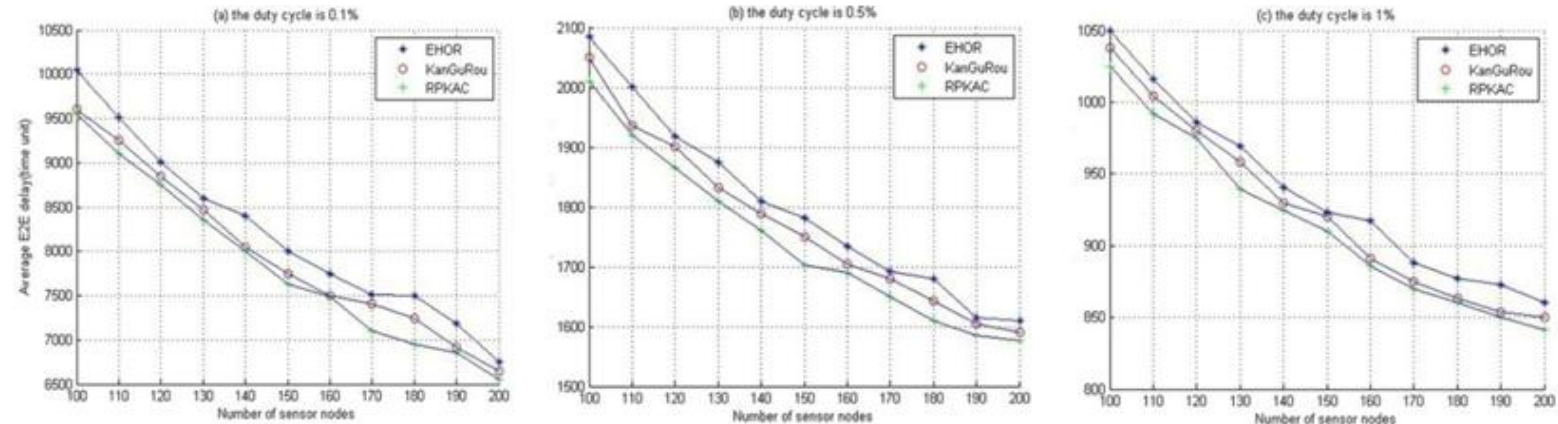

Fig7. The average lag E2E because of the node increasing number

After that, figure 7 shows the energy extraction for all sensor nodes and the considered node numbers should be four, six, 8 for k. figure 7 shows that, the energy consumption increases because of regular increasing of nodes. The energy consumption is more than other two algorithm. When the data generation nodes increases, the energy cost will modify considerably. 

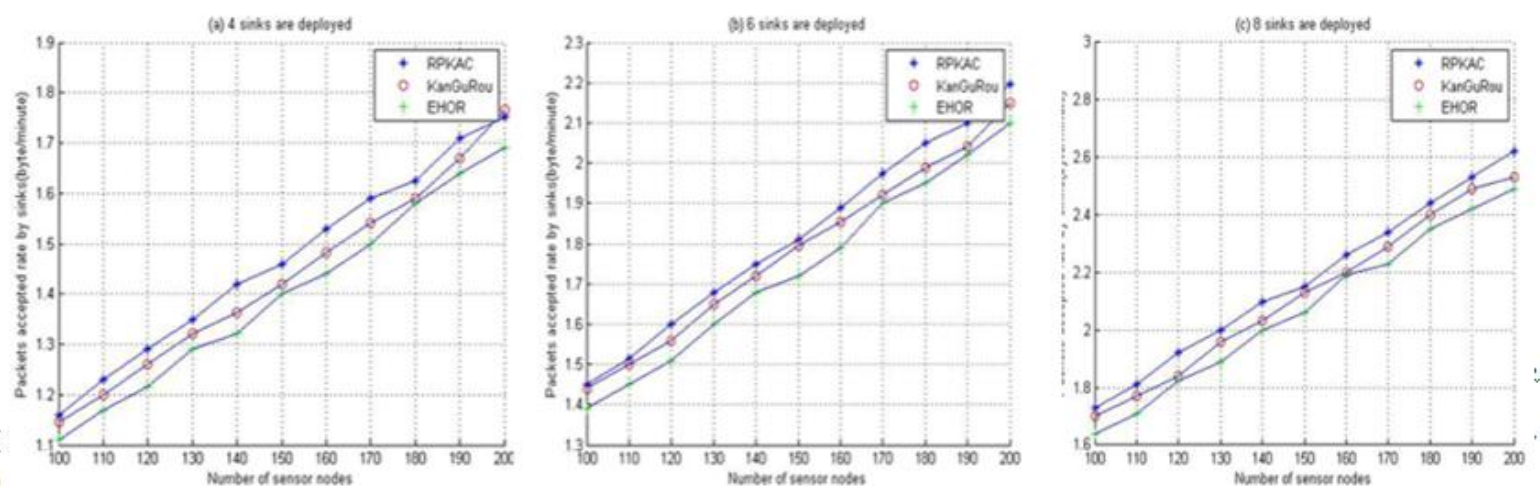

Fig8. The package receiving ability by sink along the increasing nodes.

Figure 8 shows that average lag of E2E decreases because of node number increasing. When the more number of sensors and sinks added to the network, an information package will be forwarded towards the sink.

Therefore the E2E lag will reduce significantly.

Tabel1. Three Algorithm Assessment On 180 Sensor Nodes.

\section{Conclusion}

In this study, three approach of transmission to minimum, maximum and exact $\mathrm{k}$ assessed. In this method, an information package can be sent without using of broadcast method with high energy loss. The results show the lag time reduction in successful state and energy balance keeping on node surfaces that shows the applicability of suggested algorithm for the wireless sensor networks.

[1] Yang K. Wireless Sensor Networks[M]. Springer, 2014.

[2] Razzaque M A, Bleakley C, Dobson S. Compression in wireless sensornetworks: A survey and comparative evaluation[J]. ACM Transactions on Sensor Networks (TOSN), 2013, 10(1): 5.

[3] Ganiyu R A, Arulogun O T, Okediran O O, et al. Development of a Wireless Sensor Network for Monitoring Environmental Condition on a Farmland[J]. Development, 2014, 7(3).

[4] Hackmann G, Guo W, Yan G, et al. Cyber-physical codesign of distributed structural health monitoring with wireless sensor networks[J]. Parallel and Distributed Systems, IEEE Transactions on, 2014, 25(1): 63-72.

[5] Wang J C, Lin C H, Siahaan E, et al. Mixed sound event verification on wireless sensor network for home automation[J]. Industrial Informatics, IEEE Transactions on, 2014, 10(1): 803-812.

[6] Pantazis N A, Nikolidakis S A, Vergados D D. Energy-efficient routing protocols in wireless sensor networks: A survey[J]. Communications Surveys \& Tutorials, IEEE, 2013, 15(2): 551-591.

[7] Xu X, Qian H Y, Gu Y L, et al. Ak-Anycast Routing Protocol Based on Broadcast Scheme for Wireless Sensor Network[J]. Sensor Letters, 2011, 9(4): 1566-1570.

[8] Din W I S W, Yahya S, Taib M N, et al. MAP: The new clustering algorithm based on multitier network topology to prolong the lifetime of wireless sensor network[C]//Signal Processing \& its Applications (CSPA), 2014 IEEE 10th International Colloquium on. IEEE, 2014: 173177.

[9] Yun Y S, Xia Y, Behdani B, et al. Distributed algorithm for lifetime maximization in a delay-tolerant wireless sensor network with a mobile sink[J]. Mobile Computing, IEEE Transactions on, 2013, 12(10): 19201930.

[10] He, Shibo. Chen, Jiming. Jiang, Fachang, Energy provisioning in wireless rechargeable sensor networks, IEEE Transactions on Mobile Computing, 2013, 10(12):1931-1942.

[11] Masdari, M., S.M. Bazarchi, and M. Bidaki, Analysis of secure LEACH-based clustering protocols in wireless sensor networks. Journal of Network and Computer Applications, 2013. 36(4): p. 1243-1260.

[12] Mitton, N., et al., Energy efficient k-anycast routing in multi-sink wireless networks with guaranteed delivery, in Ad-hoc, Mobile, and Wireless Networks. 2012, Springer. p. 385-398.

[13] Xu, X., et al. A distributed k-anycast routing protocol based on mobile agents. in Wireless Communications, Networking and Mobile Computing, 2009. WiCom'09. 5th International Conference on. 2009. IEEE.

[14] Frey, H., F. Ingelrest, and D. Simplot-Ryl. Localized minimum spanning tree based multicast routing with energy-efficient guaranteed delivery in ad hoc and sensor networks. in World of Wireless, Mobile and Multimedia Networks, 2008. WoWMoM 2008. 2008 International Symposium on a. 2008. IEEE.

[15] Eu,Zhi Ang,Tan, Hwee-Pink; Seah, Winston K.G. Opportunistic routing in wireless sensor networks powered by ambient energy harvesting, Computer Networks, December 3, 2010: 2943-2966.

[16] Wu, B. and J. Wu. k-Anycast routing schemes for mobile ad hoc networks. in Proceedings 20th IEEE International Parallel \& Distributed Processing Symposium. 2006. IEEE.

[17] Akyildiz, I.F., et al., A survey on sensor networks. IEEE communications magazine, 2002. 40(8): p. 102-114.

[18] Amruta M K, Satish M T. Solar powered water quality monitoring system using wireless sensor network Automation, Computing,Communication, Control and Compressed Sensing (iMac4s), 2013 International Multi-Conference on. IEEE, 2013: 281285. 
[19] Zheng, J. and A. Jamalipour, Wireless sensor networks: a networking perspective. 2009: John Wiley \& Sons.

[20] Patra, S. and J. Mallick, Secure Geographic Routing Hybrid Approach For Void Resolution In Wireless Sensor Network.

[21] Al-Karaki, J.N. and A.E. Kamal, Routing techniques in wireless sensor networks: a survey. IEEE wireless communications, 2004 11(6): p. 6-28.

[22] Forouzan, B.A., TCP/IP protocol suite. 2002: McGraw-Hill, Inc. 